\title{
Polarization variability in the interacting Be binary CX Draconis
}

\author{
A. Berdyugin and V. Piirola
}

Tuorla Observatory, 21500 Piikkiö, Finland

e-mail: andrei@astro.utu.fi;piirola@astro.utu.fi

Received 10 April 2002 / Accepted 31 July 2002

\begin{abstract}
We present new $U B V R I$ polarization measurements of the interacting Be binary CX Dra. On time scale of several weeks polarization variations clearly correlate with the binary orbital motion. Long term changes in polarization are seen in the course of several months. Analysis of the periodic component of polarization gives orbital inclination $i \simeq 73^{\circ}$ which is substantially higher than values previously reported in the literature $\left(55^{\circ}\right)$. Our two datasets obtained about 9 months apart reveal significant changes in the distribution of the scattering material of the circumbinary envelope.
\end{abstract}

Key words. stars: binaries: close - stars: emission line, Be - stars: individual: CX Dra

\section{Introduction}

CX Dra (HD 174237, HR 7084, $V=5.9$ ) is a well known Be binary with emission-line spectrum and 6.696 days orbital period. The system consists of B2.5 V primary component and F5III secondary which fills its Roche lobe. There is an accretion disk around the primary and an extended circumbinary envelope. Detailed analysis of the emission lines behaviour has given a rather complex picture of the circumstellar environment, with significant changes seen on time scales from several days to several months (see Simon 1996; Richards et al. 2000). CX Dra is a non-eclipsing variable with long-term irregular fluctuations and lower amplitude periodic residual light variations which are attributed to the proximity effect (Koubsky et al. 1980). The absence of eclipses does not allow direct determination of the sizes of the components.

Variability of polarization in CX Dra has been found by Huang et al. (1989). These authors concluded that phase-locked polarization variations synchronous with the orbital motion are superimposed on slowly varying irregular long-term changes. Lately, these data have been used by Iwamatsu et al. (2000) to make some estimates of the envelope properties. Taking into account the quantity and quality of the data, these estimates, however, can only be considered as very approximate. Our new analysis is based on much more extensive polarization data set, obtained simultaneously in five photometric passbands. We derive the orbital inclination and other important parameters with errors estimates, and present a detailed discussion of these findings.

Send offprint requests to: A. Berdyugin, e-mail: andrei@astro.utu.fi

\section{Observations}

Our polarization observations include three sets of data, first one obtained in 1997 at Crimean Astrophysical Observatory with the AZT-11 $(1.25 \mathrm{~m})$ telescope, the second and third in 2000-2001 on La Palma with the KVA $(60 \mathrm{~cm})$ telescope. Both telescopes are equipped with almost identical copies of $U B V R I$ photo-polarimeter (Piirola 1973, 1988; Korhonen et al. 1984). Polarization standard stars were also observed to determine the instrumental polarization and zero-point of polarization angle. The value of instrumental polarization for both telescopes was found to be very small $(<0.03 \%)$ except in the $U$ passband for the AZT-11 telescope $\left(P_{\text {inst }} \approx 0.2 \%\right)$. The typical error of polarization measurements for the $U B V R$ passbands is less than $0.02-0.03 \%$ and about of $0.03-0.05 \%$ for the $I$ band. The Crimean data set consists of 12 data points obtained in 18 Aug.-17 Sep. 1997. The first data set from La Palma includes 28 measurements in 14 Sep.-14 Oct. 2000, and the second set,- 19 points in 16 Jun.-4 Jul. 2001. Figure 1 shows the dependence of degree of polarization on Julian date.

\section{Polarization variability in CX Dra}

The average degree of polarization in CX Dra is rather small: $P \approx 0.2-0.4 \%$ in the $U B V$ bands and $0.1-0.3 \%$ in the $R$ and $I$ bands. The amplitude of variations also decreases noticeably towards longer wavelengths. There is no direct estimate of interstellar polarization for CX Dra, but according to Iwamatsu et al. (2000) the nearby stars show very little polarization. The binary is located at $l=82^{\circ}, b=22^{\circ}$, in a direction where interstellar polarization seems to be very low indeed. 


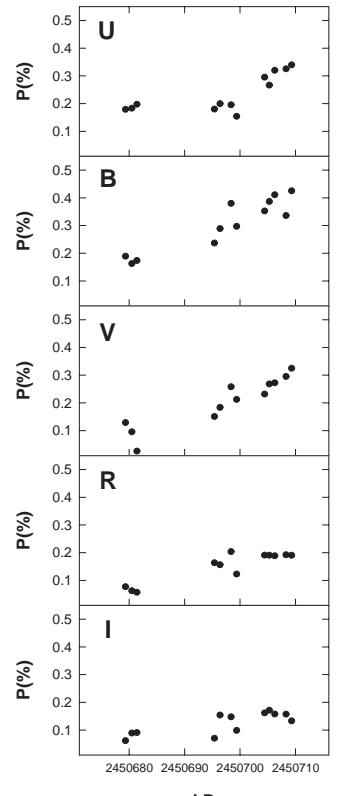

J.D.

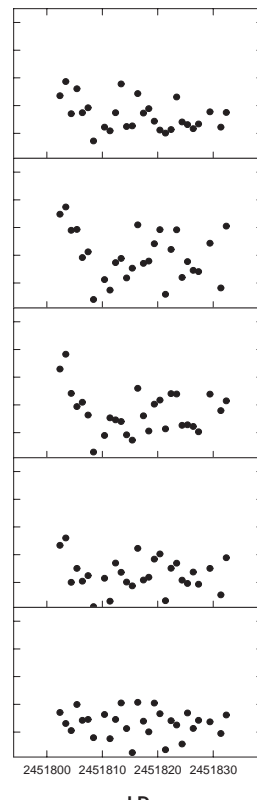

J.D.

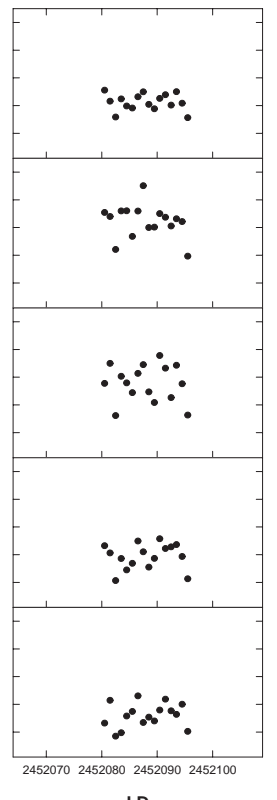

J.D.

Fig. 1. Polarization of CX Dra in the $U B V R I$ passbands plotted against Julian date for three time intervals: 1997 Aug. 18-Sep. 17 (left), 2000 Sep. 14-Oct. 14 (middle) and 2001 Jun. 16-July 4 (right).

Figure 2 shows normalized Stokes parameters of CX Dra plotted in the $(Q, U)$ plane, separately for the five passbands. Data for the three observational sets are plotted with different symbols. The points occupy well defined, rather restricted ellipse-shaped areas in the $(Q, U)$ plane, the major axis being approximately aligned with the line passing through the origin of the coordinate axes.

There is a preferred direction of orientations of observed polarization vectors in Fig. 2. If the polarization in CX Dra is due to scattering in the circumbinary envelope, this direction gives the orientation of the minor axis of the envelope projected on the plane of the sky, i.e., $\approx 70^{\circ}$ measured counter-clockwise from North.

\subsection{Wavelength dependence of polarization}

Polarization of CX Dra shows clearly maximum in the $B$ band. Variations in different passbands correlate well and the wavelength dependence of polarization remains practically unchanged from season to season, even when the average degree of polarization changes. Figure 3 shows average polarization for the three data sets, plotted as a function of the effective wavelength of the $U B V R I$ passbands. Polarization peaks around $0.4 \mu \mathrm{m}$, with a sharp drop in the $U$ band and smoother decrease towards longer wavelengths. This is a very typical feature of Be stars (Poeckert \& Marlborough 1978; Poeckert et al. 1979): the decrease of continuum polarization longward of the Balmer series limit is due to hydrogen free-free absorption of the scattered radiation in the outer parts of the envelope. Another typical feature of Be stars polarization spectrum rapid increase of polarization at Balmer series limit (Balmer "jump") cannot be directly measured with broad band photometric system like Jonson's $U B V$. However, its presence is
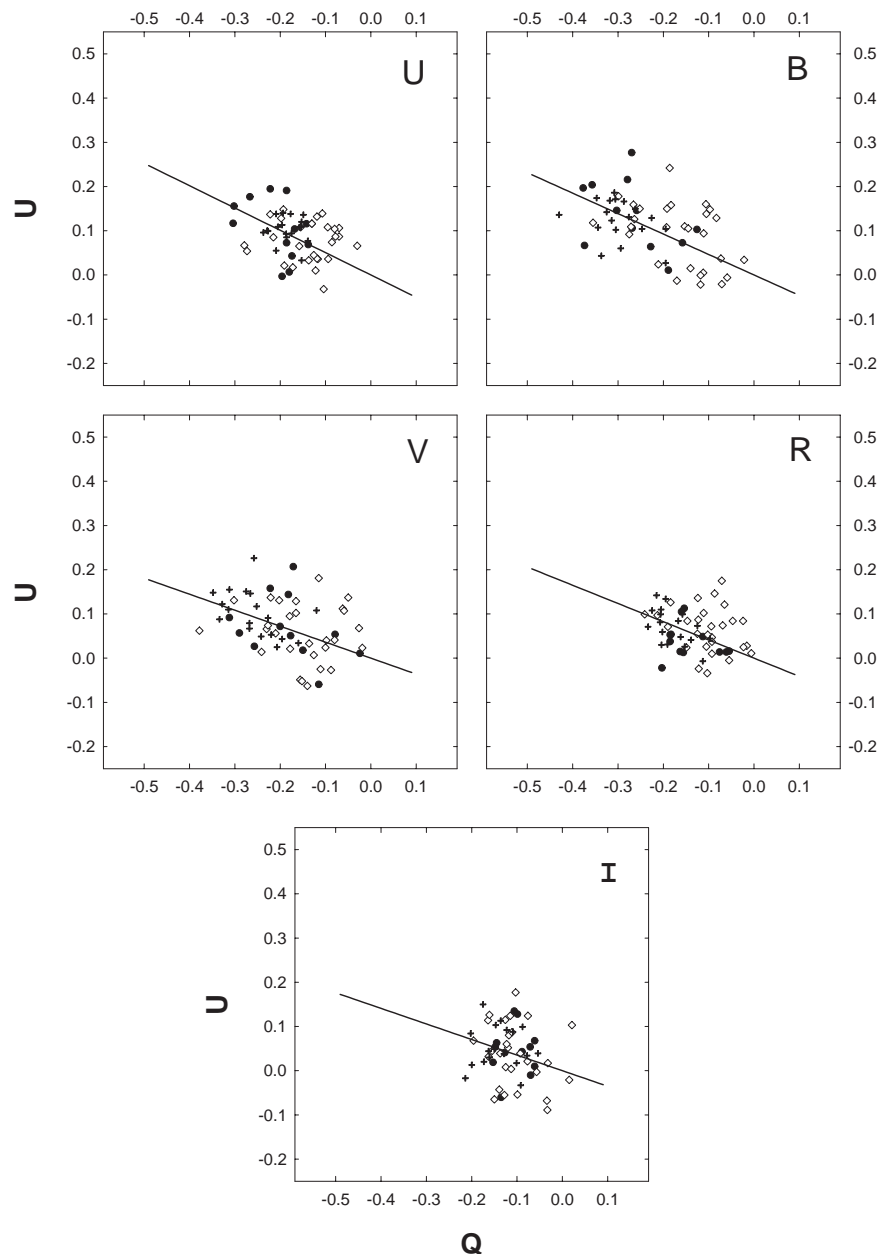

Fig. 2. Normalized Stokes parameters of CX Dra plotted in the $(Q, U)$ plane. Filled circles show the data from the first data set, empty circles from the second and crosses from the third (see Fig. 1). Sraight line is a liner regression passing through the origin of the coordinate axes.

clearly seen: in the $U$ band which overlaps Balmer "jump", polarization is substantially smaller than in the $B$ band which has effective wavelength just long-ward of it. The fact that the wavelength dependence of polarization in CX Dra, in spite of its rather small value, has the shape predicted entirely by scattering and absorption processes in the envelope, gives additional arguments in favour of negligible interstellar component.

\subsection{Phase-locked polarization variations}

In binary stars with gaseous envelopes and mass exchange, the observed polarization often varies synchronously with the orbital motion. However, in CX Dra the periodic variations have a small amplitude and are superimposed on slowly varying nonperiodic component. This long-term variability seems to be irregular in nature (Huang et al. 1989) and disturbs the cyclic pattern if data points obtained over an interval of several months are combined into one phase diagram. This complicates the detection and analysis of the periodic component. In order to resolve te problem, Huang et al. (1989) divided the observations into short runs by grouping polarization measurements obtained within a week into separate phase diagrams, which 


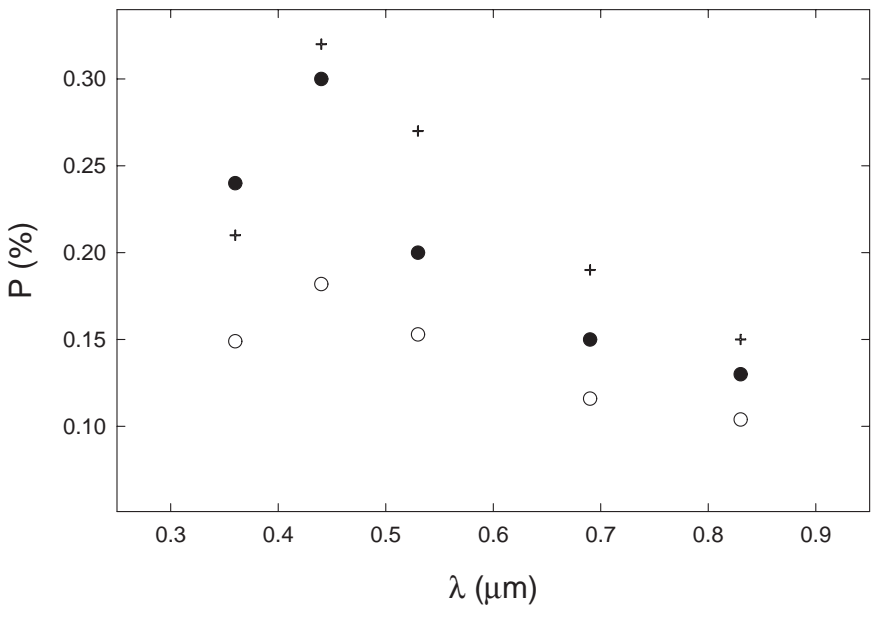

Fig. 3. Wavelength dependence of polarization of CX Dra. Filled circles show average polarization from Aug.-Sep. 1997, empty circles from Sep.-Oct. 2000, and crosses from Jun.-Jul. 2001, plotted against effective wavelength ( $U B V R I$ passbands).

were then combined together by shifting the data points in the direction of the ordinate. This method cannot yield a good and reliable phase curve as these grouping and shifting can be rather subjective, especially when small amount of polarization data (only 17 points) are collected over a period of about six months.

Our situation, however, is better as we have three separate multi-wavelength data sets $(12,28$ and 19 points), each of which is obtained within a period of about or less than one month. This allows us to analyse the periodic component in each set separately. No additional grouping or shifting of ordinates needs to be applied.

Figures 4-5 show the variations of normalized Stokes parameters of CX Dra over the orbital cycle for the La Palma data sets. The data have been phase-folded using the ephemeris from Richards et al. (2000), $-T_{\max , R V}=$ JD $2442549.48 \pm 6.695957$.

The pattern of polarization variations over the orbital cycle is clearly seen. However, we have found no clear evidence for the periodic component in our Crimean data set, though the amplitude of variations is quite high. Figure 1 shows that in this interval, 18 Aug.-17 Sep. 1997 the polarization of CX Dra increased with time rather linearly. This trend is well seen in the $U B V$ passbands where the degree of polarization doubled in two weeks.

The commonly used approach (Brown et al. 1978) is to represent the orbital phase dependence of the normalized Stokes parameters with Fourier series including first and second harmonics terms:

$q=q_{0}+q_{1} \cos (\lambda)+q_{2} \sin (\lambda)+q_{3} \cos (2 \lambda)+q_{4} \sin (2 \lambda)$,

$u=u_{0}+u_{1} \cos (\lambda)+u_{2} \sin (\lambda)+u_{3} \cos (2 \lambda)+u_{4} \sin (2 \lambda)$

where $\lambda=2 \pi \phi$ and $\phi$ is the orbital phase.

The quality of the fits for both La Palma data sets is rather good, except for the $I$ passband, where the observing errors are somewhat higher. However, even in this band the phase dependence of polarization is quite convincing. The shape of the polarization curves of CX Dra is typical for binaries with
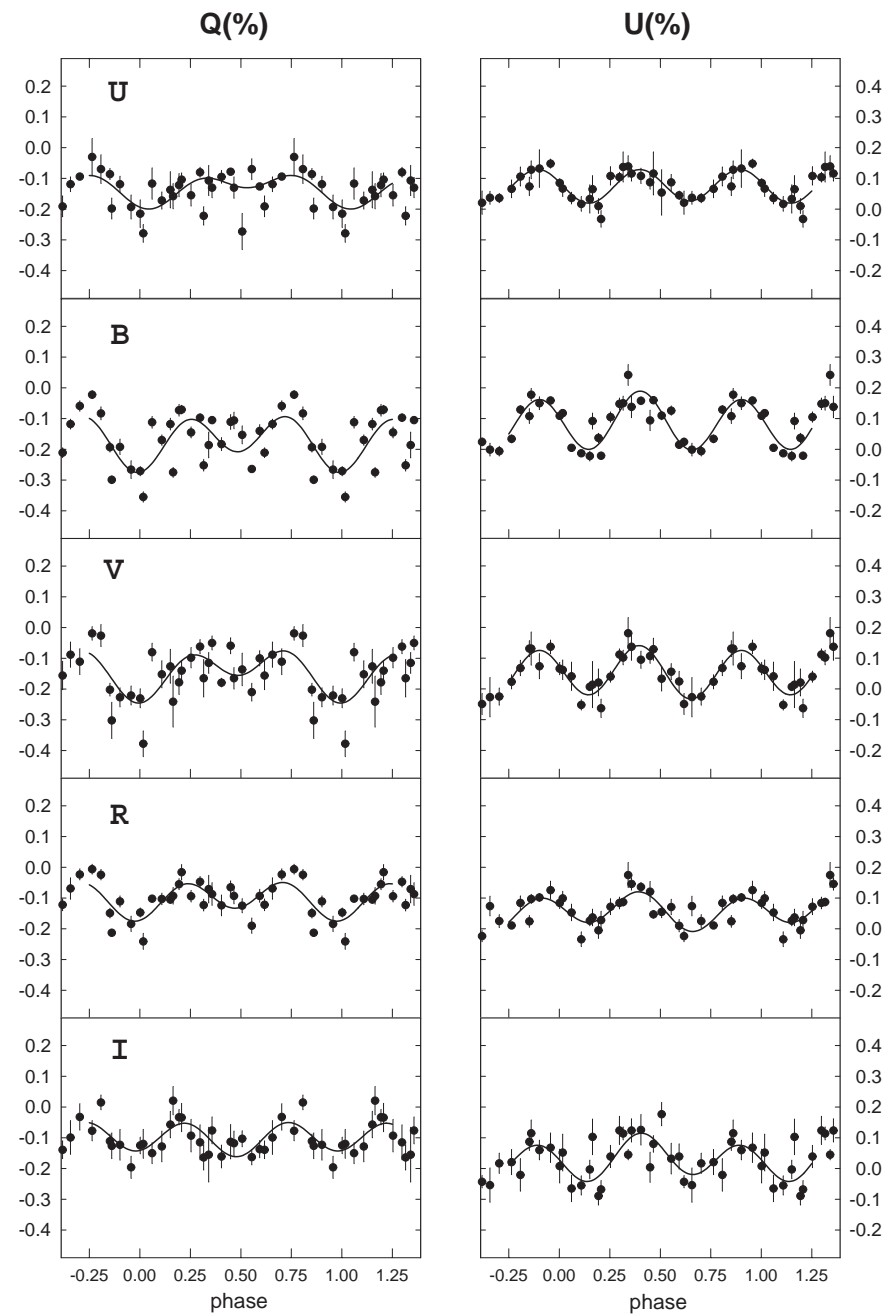

Fig. 4. Normalized Stokes parameters $Q$ and $U$ for CX Dra in 2000 Sep. 14-Oct. 14, plotted over the orbital cycle. Vertical bars are $2 \sigma$ errors. The solid lines show the two harmonics Fourier fit.

phase-locked polarization: the second harmonic clearly dominates. This is expected for envelopes which have high degree of symmetry about orbital plane. However, the polarization curves for the two La Palma data sets look different. The amplitude of variations in 2001 Jun. 16-Jul. 4 is noticeably smaller, while the average polarization is higher. Also the shape of the curves is different, with a clearly seen phase shift. This means that significant changes in the distribution of the scattering material in the envelope occurred in 9 months.

\subsection{Parameters of $C X$ Dra derived from polarimetry}

Analysis of the phase-locked polarization variations in binaries allows us to derive the orbital inclination, $i$, the orientation of orbital axis on the plane of the sky, $\Omega$, and the longitude of the light scattering region in the binary reference frame, $\lambda_{2}$. These parameters can be computed from the coefficients of the Fourier fit (see Drissen et al. 1986). Table 1 gives the parameters for the first La Palma data set, and Table 2 for the second set. Accuracy estimator $\gamma$ was used as the weight when computing the average values of $i, \Omega$ and $\lambda_{2}$ from the five 

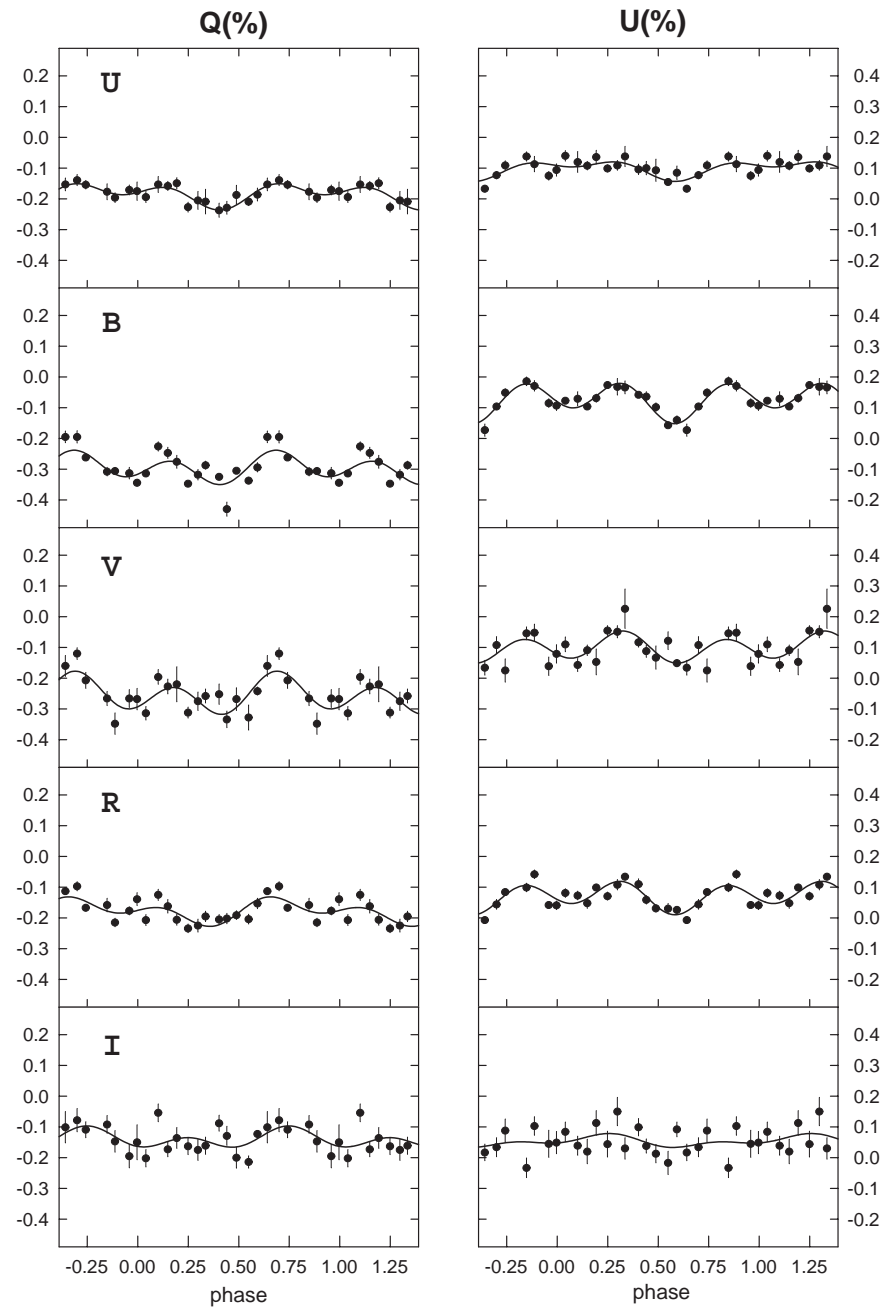

Fig. 5. Normalized Stokes parameters $Q$ and $U$ for CX Dra in 2001 Jun. 16-Jul. 4, plotted over the orbital cycle (see Fig. 4).

passbands. This estimator, according to Simmons et al. (1982) and Wolinski \& Dolan (1994) is:

$\gamma=\left(\frac{A}{\sigma_{P}}\right)^{2} \frac{N}{2}$.

Here $\sigma_{P}$ is the average error of measurement, $N$ - the number of data points, and $A$ - the amplitude of polarization variations given by:

$A=\left(\left|q_{\max }-q_{\min }\right|+\left|u_{\max }-u_{\min }\right|\right) / 4$.

Agreement between the parameters derived from the different passbands is rather good for both La Palma data sets, though it is better for the first set, where the amplitude of the variations and therefore the accuracy merit $\gamma$ is higher. For the second set the parameters from the $I$ band data are less accurate because of the very low $\gamma$. Table 3 shows the average $\bar{i}, \bar{\Omega}$ and $\bar{\lambda}_{2}$ calculated from the five passbands, and their $2 \sigma$ confidence intervals determined by applying diagrams given by Wolinski \& Dolan (1994). Confidence interval for $i$ is asymmetric (biased towards the lower inclinations) and its lower and upper borders are given in brackets. The confidence intervals for all parameters derived from the second set are broader, which is entirely due to the smaller amplitude of variations.
Table 1. $i, \Omega, \lambda_{2}$ and $\gamma$ for the first La Palma set.

\begin{tabular}{cccccc}
\hline \hline & $U$ & $B$ & $V$ & $R$ & \multicolumn{1}{c}{$I$} \\
\hline$i$ & $71^{\circ}$ & $72^{\circ}$ & $72^{\circ}$ & $76^{\circ}$ & $74^{\circ}$ \\
$\Omega$ & $78^{\circ}$ & $122^{\circ}$ & $115^{\circ}$ & $134^{\circ}$ & $124^{\circ}$ \\
$\lambda_{2}$ & $41^{\circ}$ & $26^{\circ}$ & $29^{\circ}$ & $23^{\circ}$ & $27^{\circ}$ \\
$\gamma$ & 40 & 370 & 85 & 120 & 40 \\
\hline
\end{tabular}

Table 2. $i, \Omega, \lambda_{2}$ and $\gamma$ for the second La Palma set.

\begin{tabular}{cccccc}
\hline \hline & $U$ & $B$ & $V$ & $R$ & \multicolumn{1}{c}{$I$} \\
\hline$i$ & $75^{\circ}$ & $70^{\circ}$ & $66^{\circ}$ & $81^{\circ}$ & $86^{\circ}$ \\
$\Omega$ & $155^{\circ}$ & $118^{\circ}$ & $155^{\circ}$ & $120^{\circ}$ & $23^{\circ}$ \\
$\lambda_{2}$ & $38^{\circ}$ & $52^{\circ}$ & $33^{\circ}$ & $57^{\circ}$ & $92^{\circ}$ \\
$\gamma$ & 35 & 160 & 45 & 110 & 7 \\
\hline
\end{tabular}

Table 3. Average $\bar{i}, \bar{\Omega}, \bar{\lambda}_{2}$ with $2 \sigma$ confidence intervals.

\begin{tabular}{ccc}
\hline \hline & $14.09-14.10 .00$ & $16.06-04.07 .01$ \\
\hline $\bar{i}$ & $73^{\circ}\left(65^{\circ}, 76^{\circ}\right)$ & $73^{\circ}\left(58^{\circ}, 80^{\circ}\right)$ \\
$\bar{\Omega}$ & $121^{\circ} \pm 8^{\circ}$ & $127^{\circ} \pm 15^{\circ}$ \\
$\overline{\lambda_{2}}$ & $27^{\circ} \pm 5^{\circ}$ & $50^{\circ} \pm 8^{\circ}$ \\
\hline
\end{tabular}

As seen from Table 3, the agreement between the average $i$ and $\Omega$ derived from the data obtained in the two different seasons is remarkably good. Of course, it is to be expected that inclination and orientation of the orbital axis do not change within the time intervals in concern, but the consistent values we have obtained demonstrate the reliability of the data and analysis methods applied.

It is interesting to note the significant difference in the longitude parameter $\lambda_{2}$ between the two La Palma observation runs. This shows that the position of the light scattering region, with respect to the line joining the stellar centres, has changed. The location of the scattering region indicates that it is associated with the gaseous stream, seen from the radial velocity variations of the $\mathrm{H} \alpha$ emission peak (Simon 1996; Richards et al. 2000). Our polarization data give an evidence for drift in longitude of this region during 9 months.

\subsection{Non-periodic component in polarization variability}

Figure 6 shows positions of the ellipses of the second harmonic in the $(Q, U)$ plane for the $B V R$ passbands, which have the highest accuracy of observations and the quality of Fourier fit. If only redistribution of the scattering material in the envelope of CX Dra takes place with time, one must expect that the centres of the ellipses coincide. However, a displacement of centres is clearly seen. The direction of the shift, which happened within 9 months is along the line passing through the origin of the coordinate axes. The same shift is seen in $U$ and $I$ passbands as well. This can be interpreted as being due to the presence of additional component of polarization which has changed its value between two observing runs while 
retaining the same direction. This additional component seems to be more or less constant during a single observational run.

Between the first and second La Palma observing runs the non-periodic component of polarization increased by a factor of $\approx 1.5$ (see also Fig. 3). Such an increase can be only explained by increase of the electron optical depth in the envelope and, therefore, by increase of the electron number density $n_{\mathrm{e}}$. Model calculations of continuum polarization in Be stars (Poeckert \& Marlborough 1978) show that such increase in polarization requires an increase of $n_{\mathrm{e}}$ by a facor of 3-5, if we assume that geometrical properties of envelope, i.e., the thickness, did not change. The models also predict more steep decrease of continuum polarization with wavelength when $n_{\mathrm{e}}$ increases, and this trend is clearly seen in Fig. 3.

According to Iwamatsu et al. (2000), periodic component in the polarization of CX Dra seems to increase in amplitude when the average polarization is larger. Our observations do not support this conclusion: average polarization during the second La Palma run was significantly higher than in the first one, but the amplitude of periodic variations was smaller. During the Crimean observing run the average polarization was pretty high, but the periodic component was not seen at all.

\subsection{Inclination of the orbit derived from polarimetry}

The inclination of the orbit of CX Dra obtained from polarimetry, $\sim 70^{\circ}$ is significantly higher than previously reported values in the literature $\left(50^{\circ}-55^{\circ}\right)$. Question arises how reliable are polarimetric inclination determinations.

For single Be stars polarimetric observations alone cannot be used to deduce envelope inclination, as the observed polarization depends also on the geometrical properties of the envelope (see Poeckert \& Marlborough 1978; Wood et al. 1997). However, in binary stars polarization is modulated by the orbital motion of the components, and the determination of inclination of the orbital plane is not affected by the assumed geometry of the envelope which needs not even to be symmetric about this plane.

The approach which we have used to derive the inclination from polarimetry assumes stationary co-rotating envelope. There is observational evidence (including the data we present in this paper) that the distribution of matter in the envelope of CX Dra is not static, it changes with time. According to Richards et al. (2000) noticeable changes in the circumstellar environment happen on the time scales of hundreds of days. However, each of our data sets was obtained during much shorter period, a few orbital cycles. A reasonable assumption is that no dramatic changes affecting polarization in the distribution of circumbinary material occurred in such a short interval. Furthermore, the inclination values we have derived for CX Dra from the two data sets obtained at La Palma (in five passbands) in Sep.-Oct. 2000 and in Jun.-Jul. 2001 are quite similar, despite of noticeable changes which happened in the envelope between these observational runs.

Inclinations derived from Fourier fits to observed polarization variations can be biased towards higher values, due to
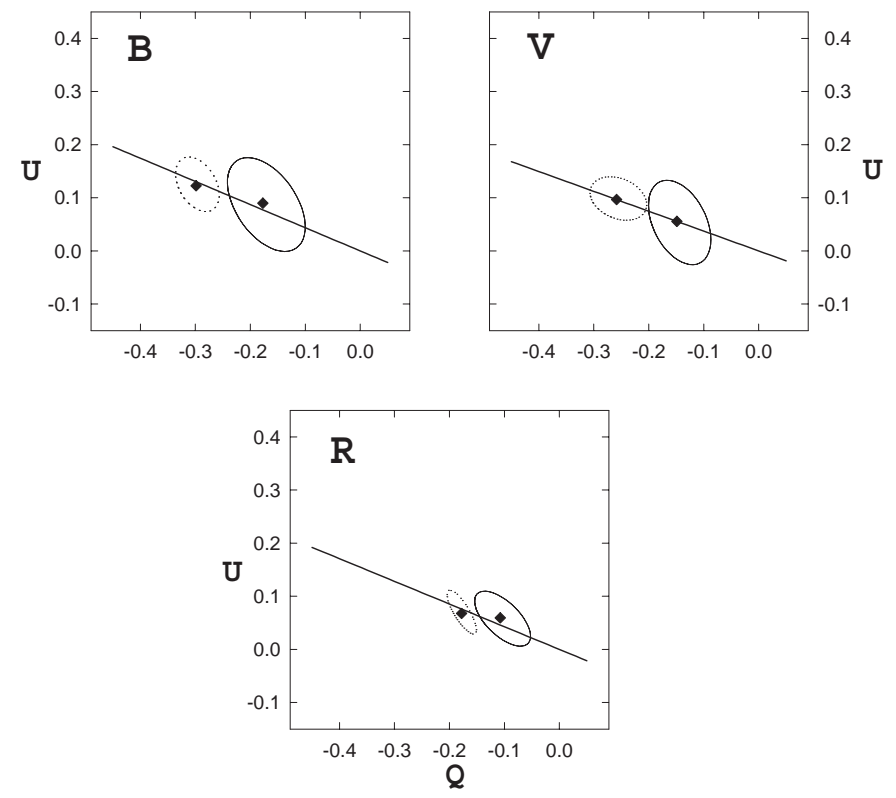

Fig. 6. Ellipses of the second harmonic of the polarization modulation over the binary orbital cycle of CX Dra are shown in the $(Q, U)$ plane for the two La Palma data sets, Sep.-Oct. 2000 (continuous line) and Jun.-Jul. 2001 (dotted line). Centers of the ellipses and the regression line passing through the origin of coordinate axes are also shown.

statistical noise in the data (Aspin et al. 1981; Simmons et al. 1982; Wolinski \& Dolan 1994). The higher the noise (the lower variability amplitude), the higher this bias. Indeed, as seen from Table 2, the lowest accuracy merit in the $I$ passband $\gamma=7$ resulted in the highest derived inclination $i=86^{\circ}$. To account for the bias which affects the dirived inclination and confidence interval we have used the method proposed by Wolinski \& Dolan (1994). We stress that our estimates are based on measurements made independently and simultaneously in five passbands which increases the statistical significance of the inferred inclination and other parameters.

\section{Effect of higher inclination on the binary parameters}

The absence of eclipses in CX Dra requires that

$\operatorname{tg} i<\frac{a_{1} \sin i+a_{2} \sin i}{R_{1}+R_{2}}$

where $i$ is the orbital inclination, $a_{1}$ and $a_{2}$ are the major semiaxes of the orbits, and $R_{1}$ and $R_{1}$ the radii of the components. If we take $a_{1} \sin i=4.5 R_{\odot}$ and $a_{2} \sin i=19.3 R_{\odot}$ from Richards et al. (2000) and assume $i=70^{\circ}$ we get $R_{1}+R_{2}<8.7 R_{\odot}$. If the primary component is a normal $\mathrm{B} 2.5 \mathrm{~V}$ star, then $R_{1} \simeq 4 R_{\odot}$ (see Harmanec 1988). The spectral type of the secondary has been estimated to be mid-F giant (Horn et al. 1992). "Typical" size of a normal $\mathrm{F}$ giants is somewhat uncertain, but generally it is assumed to be in the range $4-5 R_{\odot}$. These size estimates are consistent with no eclipses taking place at inclination as high as $\simeq 70^{\circ}$.

The inclination $\simeq 70^{\circ}$ would lead to smaller masses of the primary and secondary components: $3.9 M_{\odot}$ and $0.9 M_{\odot}$ 
respectively. These masses are too low for their earlier accepted spectral types, and correspond to B5.5 V primary with $T_{\text {eff }} \simeq 15000 \mathrm{~K}$, and a secondary which is too light to be a normal F giant. We must note, however, that CX Dra is an interacting binary with intense and non-stationary mass exchange. Therefore, the components cannot be considered as normal stars, as their evolution has been influenced by the mass transfer. We also point out that our estimate of the inclination was derived directly from polarization data without any assumptions about physical properties of the components. Taking into account the $2 \sigma$ confidence intervals given in Table 3 , inclinations higher than $60^{\circ}$ should be considered seriously in future models of this binary system.

\section{Conclusions}

Our polarimetric investigation of CX Dra has revealed a complex picture of variability: the pattern of orbitally phase-locked polarization variations changes on time scales of months, and is superimposed onto another polarization component, slowly varying in the course of several months to years. The variations in the long-term component can be explained by changes in the overall optical depth of the envelope, while the variations in the pattern of the periodic component are due to the redistribution of the light scattering material in the orbital reference frame. Our observations give evidence of a noticeable drift in the longitude of the region associated with the mass stream from the secondary to the primary, which took place in 9 months. Our analysis of the phase-locked polarization variations also suggests orbital inclination value $\left(i \sim 70^{\circ}\right)$ which is substantially higher than previously reported in the literature $\left(50^{\circ}-55^{\circ}\right)$.

\section{References}

Aspin, C., Simmons, J. F. L., \& Brown, J. C. 1981, MNRAS, 194, 283

Brown, J. C., McLean, I. C., \& Emslie, A. G. 1978, A\&A, 68, 415

Drissen, L., Lamontagne, R., Moffat, A. F. J., et al. 1986, ApJ, 304, 188

Simon, V. 1996, A\&A, 308, 799

Richards, M., Koubsky, P., Simon, V., et al. 2000, ApJ, 531, 1003

Harmanec, P. 1988, Bull. Astron. Inst. Czech., 39, 329

Horn, J., Hubert, A. M., Hubert, H., et al. 1992, A\&A, 259, L5

Koubsky, P., Harmanec, P., Horn, P., et al. 1980, Bull. Astron. Inst. Czech., 31, 75

Huang, L., Hsu, J. C., \& Guo, Z. H. 1989, A\&AS, 78, 431

Iwamatsu, H., Hirata, R., \& Masuda, S. 2000, in The Be Phenomenon In Early-Type Stars, IAU Coll. 175, ed. M. Smith, H. F. Henrichs, \& J. Fabregat, ASP Conf. Ser., 214

Korhonen, T., Piirola, V., \& Reiz, A. 1984, ESO Messenger, No 38

Piirola, V. 1973, A\&A, 27, 383

Piirola, V. 1988, in Polarized Radiation of Circumstellar Origin, ed. G. V. Coyne, A. F. J. Moffat, S. Tapia, et al. (Tucson, Univ. of Arizona, Press), 735

Poeckert, R., \& Marlborough, J. M. 1978, ApJS, 38, 229

Poeckert, R., Bastien, P., \& Landstreet, J. D. 1979, AJ, 84, 812

Simmons, J. F. L., Aspin, C., \& Brown, J. C. 1982, MNRAS, 198, 45

Wolinski, K. G., \& Dolan, J. F. 1994, MNRAS, 267, 5

Wood, K., Bjorkman, K. s., \& Bjorkman, J. E. 1997, ApJ, 477, 926 